\title{
Prediction of progression of damage to articular cartilage 2 years after anterior cruciate ligament reconstruction: use of aggrecan and type II collagen biomarkers in a retrospective observational study
}

Yasumori Sobue ${ }^{1}$, Toshihisa Kojima ${ }^{1 *}$, Kazutoshi Kurokouchi², Shigeo Takahashi², Hiroaki Yoshida ${ }^{3}$, Robin Poole ${ }^{4}$ and Naoki Ishiguro ${ }^{1}$

\begin{abstract}
Background: We aimed to determine whether synovial fluid (SF) biomarkers can predict the progression of articular cartilage damage as determined by arthroscopic evaluation during and after anterior cruciate ligament $(\mathrm{ACL})$ reconstruction.

Methods: Arthroscopic assessment of articular cartilage damage was performed twice in 62 patients, first during $A C L$ reconstruction and then approximately 2 years later during implant removal for ligament fixation. SF levels of the collagenase-generated cleavage neoepitope of type II collagen (C2C) and proteoglycan glycosaminoglycans keratan sulfate (KS), chondroitin-4-sulfate ( $\Delta$ di-C4S), and chondroitin-6-sulfate ( $\Delta$ di-C6S) were measured at ACL reconstruction. Associations between baseline biomarker levels and subsequent progression of cartilage damage were determined using receiver operating characteristic analysis and multivariable logistic regression analysis.

Results: No radiographic changes were observed in any of the patients. Progression of high-grade cartilage damage, observed arthroscopically, was negatively correlated with levels of $\Delta$ di-C6S and KS, as well as the ratio of $\Delta$ di-C6S to $\Delta$ di-C4S (C6S/C4S). Logistic regression analysis revealed significant associations of $\Delta$ di-C6S (cut-off: $55.7 \mathrm{nmol} / \mathrm{ml}$, odds ratio (OR) 0.231, 95\% confidence interval (Cl) 0.061-0.879), KS (cut-off: $10.6 \mu \mathrm{g} / \mathrm{ml}$, OR 0.114, 95\% Cl 0.024-0.529), and C6S/C4S ratio (cut-off: 4.6, OR 0.060, 95\% Cl 0.005-0.737) with the progression of high-grade cartilage damage after adjusting for age, the duration from injury to first surgery, sex, and the number of high-grade lesions (grades III and IV) at baseline.
\end{abstract}

Conclusions: The progression of high-grade cartilage damage was significantly associated with baseline levels of proteoglycan glycosaminoglycan biomarkers; namely, $\Delta$ di-C6S, KS, and C6S/C4S ratio.

Keywords: Cartilage, Biomarker, Anterior cruciate ligament injury, Keratan sulfate, Chondroitin sulfate, Arthroscopy

\footnotetext{
* Correspondence: toshik@med.nagoya-u.ac.jp

${ }^{1}$ Department of Orthopedic Surgery, Nagoya University School of Medicine,

65 Tsurumai, Showa, Nagoya 466-8550, Japan

Full list of author information is available at the end of the article
} 


\section{Background}

Destruction of the articular cartilage matrix is one characteristic of osteoarthritis (OA), which can be associated with intolerable pain and difficulties in daily life such as walking, going up and down stairs, and other movements accompanying flexion and extension of the knee. Progression of cartilage damage cannot be prevented once radiographic changes appear. Therefore, detection of early cartilage damage is necessary in order to prevent OA progression.

The articular cartilage matrix is mainly composed of type II collagen and proteoglycans such as aggrecan. Collagen fibrils provide tensile strength to maintain tissue integrity, whereas aggrecan, interwoven with collagen fibrils, contributes to compressive stiffness [1]. Excessive cleavage of type II collagen by collagenases is thought to represent a critical step leading to the destruction of the cartilage matrix. In fact, the collagenasegenerated cleavage neoepitope of type II collagen (C2C) is a useful biomarker that reflects the cleavage of type II collagen in patients suffering from anterior cruciate ligament (ACL) injury [2, 3] or OA [4, 5]. Aggrecan is the major cartilage proteoglycan. It consists of a core protein to which glycosaminoglycan side chains of keratan sulfate (KS) and chondroitin sulfate (CS) are attached $[6,7]$. Chondroitin-6-sulfate $(\Delta$ di-C6S) and KS are most abundantly found in normal adult articular cartilage, where they are replaced with chondroitin-4sulfate $(\triangle \mathrm{di}-\mathrm{C} 4 \mathrm{~S})$ of newly synthesized proteoglycan as cartilage degradation progresses [8-10].

We recently reported that synovial fluid (SF) C2C and KS biomarkers are associated with the degree of cartilage damage based on arthroscopic evaluation in patients with ACL injury [1]. The number of high-grade cartilage lesions was associated with levels of $\mathrm{C} 2 \mathrm{C}$ and $\mathrm{KS}$. Moreover, our findings suggested that a combination of higher $\mathrm{C} 2 \mathrm{C}$ and lower KS may offer a greater ability to identify patients with pre-radiographic, early high-grade cartilage damage compared to a single clinical or biomarker parameter.

Given the importance of longitudinal studies in clarifying the value of biomarkers to detect progression of cartilage damage before radiographic changes appear, this study aimed to investigate whether the progression of cartilage damage after ACL reconstruction was related to SF biomarkers measured at the time of ACL reconstruction, and whether they could predict progression of cartilage damage based on longitudinal arthroscopic evaluation.

\section{Methods}

\section{Patients}

Among 108 patients with ACL injury who participated in our previous study [1], 92 and 16 underwent ACL reconstruction between January 2001 and March 2003 at Mitsubishi Nagoya Hospital (Nagoya, Japan) and Kasugai
Orthopedic Hospital (Kasugai, Japan) respectively. Reconstruction of the ACL was performed using the hamstring tendon with femoral fixation by the EndoButton CL device (Smith \& Nephew Endoscopy, Andover, MA, USA) and tibial fixation by staples.

Of the 108 patients, 62 were followed and underwent two arthroscopic evaluations, one during ACL reconstruction (baseline) and another upon implant (staples) removal for tibial fixation of the reconstructed ligament approximately 2 years post-operatively (follow-up). All 62 patients who participated in this study were cases of Mitsubishi Nagoya Hospital. Because the staples are felt subcutaneously and cause pain or a feeling of alien substance, they are removed in most cases. Reasons for patient drop-out from our follow-up study were unclear, but there were no significant differences in patient baseline characteristics except for KS levels between the 62 patients in this study and the other remaining 46 patients (Additional file 1).

The study was approved by the Ethics Committee of Nagoya University School of Medicine (2017-0140). This is retrospective research including the information on synovial fluid obtained from the previous study. Therefore, we disclosed the study information at the site of the related facilities instead of using a consent form according to committee procedure.

Arthroscopic evaluations of articular cartilage and menisci As was done in our earlier study [1], articular cartilage damage was evaluated at six sites (Fig. 1), including the patella, femoral groove, lateral femoral condyle, medial femoral condyle, lateral tibial plateau, and medial tibial plateau. We again used the Outerbridge grading system [11], which assesses both the depth and size of macroscopic chondropathic changes involving the articular cartilage surface in a range of grade 0 (normal) to IV. Grade I is characterized by softening and swelling of the cartilage. Grade II reflects the presence of macroscopically observable fragmentation and fissuring in an area $1.27 \mathrm{~cm}$ or less in diameter. Grade III represents fragmentation and fissuring that occupy an area more than $1.27 \mathrm{~cm}$ in diameter. Grade IV is characterized by erosion of cartilage with eburnation [1]. The meniscal condition was also recorded. A complete meniscal tear or defect involving more than half of the meniscus, including meniscectomy status, was defined as high-grade meniscus damage. The mean grade from three evaluators was recorded both at ACL reconstruction (baseline) and implant removal (follow-up). Inter-observer error was found to be $9.1 \%$ [1].

\section{Clinical evaluation of $A C L$ reconstruction}

Results of ACL reconstruction were evaluated by radiographic examination, using the Tegner activity level scale 


\begin{tabular}{|c|c|c|c|c|c|}
\hline \multirow[b]{2}{*}{$\%$} & \multirow[b]{2}{*}{ Progress } & \multirow{2}{*}{$\begin{array}{c}\text { Patella } \\
\text { Non-progress }\end{array}$} & \multicolumn{2}{|c|}{ Femoral groove } & \multirow[b]{2}{*}{ Non-progress } \\
\hline & & & $\%$ & Progress & \\
\hline baseline & 13.3 & 6.4 & baseline & 6.7 & 6.4 \\
\hline follow-up & 26.7 & 6.4 & follow-up & 53.3 & 10.6 \\
\hline \multicolumn{3}{|c|}{ Lateral femoral condyle } & \multicolumn{2}{|c|}{ Medial femoral condyle } & \\
\hline$\%$ & Progress & Non-progress & $\%$ & Progress & Non-progress \\
\hline baseline & 13.3 & 8.5 & baseline & 13.3 & 21.3 \\
\hline follow-up & 40.0 & 2.1 & follow-up & 20.0 & 8.5 \\
\hline \multicolumn{3}{|c|}{ Lateral tibial plateau } & \multicolumn{2}{|c|}{ Medial tibial plateau } & \\
\hline$\%$ & Progress & Non-progress & $\%$ & Progress & Non-progress \\
\hline baseline & 20.0 & 8.5 & baseline & 0 & 4.3 \\
\hline follow-up & 53.3 & 8.5 & follow-up & 6.7 & 4.3 \\
\hline
\end{tabular}

Fig. 1 Proportions (\%) of high-grade cartilage damage in progression and non-progression groups at baseline and follow-up. Arthroscopic grading was performed on each of six articular sites (as shown) according to the Outerbridge scoring system. High-grade cartilage damage was defined as Outerbridge grades III and IV. Baseline, ACL reconstruction; follow-up, implant removal; progression group, patients with an increase (between baseline and follow-up) in the number of high-grade cartilage lesions (Outerbridge grades III and IV) by one score in any of the six compartments; non-progression group, patients with no increase in the number of high-grade cartilage lesions

and the pivot shift test. Knee radiographs were examined and graded according to the Kellgren-Lawrence scale [12]. Higher than grade I was defined as radiographic OA. The Tegner activity level scale provides numerical grades for work and sport activities and assesses disability after knee ligament injury [13]. The pivot shift test assesses knee stability, particularly with regard to rotatory instability after reconstruction [14].

\section{Sampling of SF and biomarker measurements}

Sampling was performed at baseline, the time of ACL reconstruction prior to arthroscopic evaluation using a lateral suprapatellar approach. Samples were centrifuged at $10,000 \mathrm{rpm}$ for $20 \mathrm{~min}$ at $4{ }^{\circ} \mathrm{C}$ and the cell-free supernatants were stored at $-80{ }^{\circ} \mathrm{C}$ until analyzed [1]. $\mathrm{C} 2 \mathrm{C}, \mathrm{KS}$, $\Delta$ di-C4S, and $\Delta$ di-C6S levels in SF samples were measured by ELISA $(\mathrm{C} 2 \mathrm{C})$ or high-performance liquid chromatography (KS, $\Delta$ di-C4S, and $\Delta$ di-C6S), as previously described [1]. Moreover, in consideration of our previous finding that the ratio of $\mathrm{C} 2 \mathrm{C}$ to $\mathrm{KS}(\mathrm{C} 2 \mathrm{C} / \mathrm{KS})$ may identify patients with early cartilage damage [1], or the reports that the ratio of $\Delta$ di-C6S to $\Delta$ di-C4S $(\mathrm{C} 6 \mathrm{~S} / \mathrm{C} 4 \mathrm{~S})$ was associated with age, sex, and the progression of OA $[15,16]$, we added $\mathrm{C} 2 \mathrm{C} / \mathrm{KS}$ and $\mathrm{C} 6 \mathrm{~S} / \mathrm{C} 4 \mathrm{~S}$ to the analyses.

\section{Progression of cartilage damage}

We defined grade III and IV lesions as high-grade cartilage damage [1]. Grade III encompasses a relatively wide range of cartilage damage and is easily distinguishable from grade II. Changes in the number of high-grade lesions (grades III and IV) from the first examination (baseline) to the second examination (follow-up) at the six articular sites were measured. The increased number of high-grade lesions (grades III and IV) indicated that the cartilage damage had increased and was approaching that observed in radiographic OA. Hence, progression of cartilage damage was defined as an increased number of high-grade cartilage lesions (grades III and IV). Of the 62 patients who were followed in this study, 15 exhibited progression of cartilage damage (progression group).

\section{Statistical analysis}

All statistical analyses were performed with EZR (Saitama Medical Center, Jichi Medical University, Saitama, Japan), a graphical user interface for $\mathrm{R}$ (The $\mathrm{R}$ Foundation for Statistical Computing, Vienna, Austria) [17]. $P<0.05$ was considered statistically significant.

Student's $t$ test was used to analyze continuous variables. Fisher's exact test was used to analyze ordinal variables and categorical variables. When comparing levels of biomarkers between progression and non-progression groups, the nonparametric Mann-Whitney $U$ test was used.

Receiver operating characteristic (ROC) curves were generated to assess associations between levels of biomarkers and progression of high-grade cartilage damage. The best cut-off point was identified as the maximum point of the Youden index, which was calculated using the following formula: Youden index = sensitivity + specificity -1 .

Finally, multivariable logistic regression analyses were performed to confirm the independent impact of variables on the progression of high-grade cartilage damage. In order to dichotomize values, cut-off points based on ROC curves were used.

\section{Results}

Lesion onset and progression

Table 1 summarizes the characteristics of patients by group (progression group, $\mathrm{n}=15$; non-progression group, 
Table 1 Characteristics of patients in the progression group and the non-progression group at baseline and follow-up

\begin{tabular}{|c|c|c|c|c|c|}
\hline Variables & & $\begin{array}{l}\text { Total } \\
(n=62)\end{array}$ & $\begin{array}{l}\text { Progression group } \\
(n=15)\end{array}$ & $\begin{array}{l}\text { Non-progression group } \\
(n=47)\end{array}$ & $p$ value \\
\hline \multicolumn{6}{|l|}{ Baseline } \\
\hline Age (years) & Mean (SD) & $27.6(9.0)$ & $31.1(9.2)$ & $26.5(8.7)$ & 0.083 \\
\hline Duration from injury to $A C L$ reconstruction (months) & Mean (SD) & $26.1(37.8)$ & $39.7(49.8)$ & $21.8(32.6)$ & 0.111 \\
\hline Sex, female (\%) & & 38.7 & 40 & 38.3 & 1 \\
\hline High-grade meniscus damage (\%) & & 58.1 & 66.7 & 55.3 & 0.553 \\
\hline More than one grade III or IV lesion (\%) & & 35.5 & 46.7 & 31.9 & 0.359 \\
\hline \multicolumn{6}{|l|}{ Follow-up } \\
\hline More than grade I of Kellgren-Lawrence (\%) & & 0 & 0 & 0 & 1 \\
\hline Duration from reconstruction to removal (months) & Mean (SD) & $26.0(7.8)$ & $27.3(11.7)$ & $25.6(6.2)$ & 0.482 \\
\hline BMI & Mean (SD) & $24.1(3.1)$ & $23.9(2.9)$ & $24.2(3.2)$ & 0.744 \\
\hline Tegner activity level scale & Mean (SD) & $5.1(2.2)$ & $5.1(2.5)$ & $5.1(2.1)$ & 0.944 \\
\hline Pivot shift test, positive (\%) & & 18.3 & 6.7 & 22.2 & 0.262 \\
\hline High-grade meniscus damage (\%) & & 64.5 & 60.0 & 68.1 & 0.755 \\
\hline More than one grade III or IV lesion (\%) & & 40.3 & 100 & 21.3 & $<0.001$ \\
\hline
\end{tabular}

Baseline, ACL reconstruction; Follow-up, implant removal; Progression group, patients with an increase (between baseline and follow-up) in the number of highgrade cartilage lesions (Outerbridge grades III and IV) by one score in any of the six compartments; Non-progression group, patients with no increase in the number of high-grade cartilage lesions; BMI body mass index; high-grade meniscus damage, complete meniscal tear or defect in more than half the meniscus; SD standard deviation. $P<0.05$ was considered statistically significant

$\mathrm{n}=47)$. The mean age was higher in the progression group (31.1 years) than in the non-progression group (26.5 years). The mean duration from injury to ACL reconstruction was longer in the progression group than in the non-progression group. There were almost no differences between groups in terms of proportion of females, proportion of high-grade meniscus damage, or proportion with more than one grade III lesion.

Patient characteristics at follow-up are also shown in Table 1. There were no patients with radiographic OA (more than grade I Kellgren-Lawrence) that showed joint space narrowing, osteophytes, and subchondral and sclerotic bone lesions. There were almost no differences between progression and non-progression groups in the duration from first to second evaluation, BMI, Tegner activity level scale, proportion of those with a positive pivot shift test, and proportion of those with high-grade meniscus damage. The proportion of those with more than one Grade III lesion at the second evaluation was higher in the progression group (100\%) than in the nonprogression group (21.3\%).

Figure 1 shows the proportions of high-grade cartilage damage in the progression and non-progression groups at baseline and follow-up. At follow-up, more than half of those in the progression group had high-grade damage at the femoral grove and lateral tibial plateau.

Table 2 shows changes in the number of grade 0-IV lesions from baseline to follow-up. In both groups, the number of grade I lesions was higher at baseline. In the progression group, the numbers of grade 0 (normal cartilage), grade I, and grade II lesions that progressed to high-grade lesions were 4, 6 , and 10, respectively: not only well-established lesions but also normal cartilage and lesions showing minimal damage exhibited development and progression of cartilage damage, respectively, based on arthroscopic evaluation. In contrast, in the non-progression group, almost all low-grade lesions remained unchanged, while some of high-grade lesions changed to low-grade lesions $(\mathrm{n}=11)$.

\section{Biomarker evaluation}

With regard to biomarkers of SF at the time of ACL reconstruction (baseline), levels of $\triangle \mathrm{di}-\mathrm{C} 6 \mathrm{~S}$ and $\mathrm{KS}$, as well as the ratio of $\Delta \mathrm{di}-\mathrm{C} 6 \mathrm{~S}$ to $\Delta \mathrm{di}-\mathrm{C} 4 \mathrm{~S}(\mathrm{C} 6 \mathrm{~S} / \mathrm{C} 4 \mathrm{~S})$, were significantly lower in the progression group compared to the non-progression group. There were no differences in levels of $\mathrm{C} 2 \mathrm{C}, \Delta$ di-C4S and the ratio of $\mathrm{C} 2 \mathrm{C}$ to $\mathrm{KS}$ $(\mathrm{C} 2 \mathrm{C} / \mathrm{KS})$ between the two groups (Table 3 ).

Using ROC analysis, we assessed the associations between the progression of high-grade cartilage damage and baseline levels of $\Delta \mathrm{di}-\mathrm{C} 6 \mathrm{~S}, \Delta \mathrm{di}-\mathrm{C} 4 \mathrm{~S}$ and $\mathrm{KS}$ as well as the $\mathrm{C} 6 \mathrm{~S} / \mathrm{C} 4 \mathrm{~S}$ ratio (Fig. 2). The areas under the ROC curve were 0.746 (95\% CI 0.612-0.880) for $\Delta$ di-C6S, 0.618 (95\% CI $0.478-0.758)$ for $\Delta$ di-C4S, 0.699 (95\% CI $0.526-$ 0.872 ) for KS, and 0.689 (95\% CI $0.546-0.833$ ) for C6S/ C4S. Best cut-off values were $55.7 \mathrm{nmol} / \mathrm{ml}$ for $\Delta$ di-C6S (sensitivity $60.0 \%$, specificity $83.0 \%$ ), $19.0 \mathrm{nmol} / \mathrm{ml}$ for $\Delta$ di-C4S (sensitivity 93.3\%, specificity $46.8 \%$ ). $10.6 \mu \mathrm{g} / \mathrm{ml}$ for KS (sensitivity $73.3 \%$, specificity $70.2 \%$ ), and 4.6 for C6S/C4S (sensitivity $93.3 \%$, specificity $40.0 \%$ ).

Table 4 shows the associations of $\Delta$ di-C6S levels, $\Delta$ diC4S levels, KS levels, and the C6S/C4S ratio with the 
Table 2 Number of grade 0-IV lesions from baseline to follow-up in the progression and the non-progression groups

\begin{tabular}{|c|c|c|c|c|c|c|c|c|c|}
\hline \multicolumn{5}{|c|}{ Progression group } & \multicolumn{5}{|c|}{ Non-progression group } \\
\hline \multirow[t]{2}{*}{ Baseline } & & & \multicolumn{2}{|l|}{ Follow-up } & \multirow[t]{2}{*}{ Baseline } & & & \multicolumn{2}{|l|}{ Follow-up } \\
\hline & & & Grade 0-II & Grade III-IV & & & & Grade 0-II & Grade III-IV \\
\hline Grade 0 & $28.9 \%$ & $(n=26)$ & $22(84.6 \%)$ & $4(15.4 \%)$ & Grade 0 & $28.7 \%$ & $(n=81)$ & 81 (100\%) & $0(0 \%)$ \\
\hline Grade I & $33.3 \%$ & $(n=30)$ & $24(80.0 \%)$ & $6(20.0 \%)$ & Grade I & $44.3 \%$ & $(n=125)$ & $122(97.6 \%)$ & $3(2.4 \%)$ \\
\hline Grade II & $26.7 \%$ & $(n=24)$ & $14(58.3 \%)$ & $10(41.7 \%)$ & Grade II & $17.7 \%$ & $(n=50)$ & 49 (98.0\%) & $1(2.0 \%)$ \\
\hline Grade III-IV & $11.1 \%$ & $(n=10)$ & $0(0 \%)$ & $10(100 \%)$ & Grade III-IV & $9.2 \%$ & $(n=26)$ & $11(42.3 \%)$ & $15(57.7 \%)$ \\
\hline
\end{tabular}

$\mathrm{N}=15$ (progression group), $\mathrm{N}=47$ (non-progression group). Total compartments that were investigated arthroscopically for the Outerbridge grading $=6$ per knee (total of $90(6 \times 15)$ compartments for the progression group and $282(6 \times 47)$ compartments for the non-progression group). Baseline, ACL construction; Follow-up, implant removal; Progression group, patients with an increase (between baseline and follow-up) in the number of high-grade cartilage lesions (Outerbridge grades III and IV) by one score in any of the six compartments; Non-progression group, patients with no increase in the number of high-grade cartilage lesions

progression of high-grade cartilage damage as revealed by logistic regression analyses. Even after adjusting for age, the duration from injury to ACL reconstruction, sex, and the number of high-grade lesions (grades III and IV) at baseline, associations remained significant between the progression of high-grade cartilage damage and levels of $\Delta$ di-C6S (OR 0.231, 95\% CI 0.061-0.879), levels of $\Delta$ di-C4S (OR 0.081, 95\% CI 0.008-0.763), KS (OR 0.114, 95\% CI 0.024-0.529), and the ratio of C6S/ C4S (OR 0.060, 95\% CI 0.005-0.737).

\section{Discussion}

We found that SF levels of $\Delta$ di-C6S, $\Delta$ di-C4S, KS and the ratio of $\Delta \mathrm{di}-\mathrm{C} 6 \mathrm{~S}$ to $\Delta \mathrm{di}-\mathrm{C} 4 \mathrm{~S}(\mathrm{C} 6 \mathrm{~S} / \mathrm{C} 4 \mathrm{~S})$ are significantly associated with the onset and progression of preradiographic high-grade focal cartilage damage-which was not reflected by a transition to detectable radiographic changes-based on longitudinal arthroscopic and radiographic evaluations. Many studies have reported on biomarkers associated with cartilage damage progression with regard to radiographic changes [18-23]. This study is the first to report that biomarkers of SF can predict preradiographic onset and progression of early cartilage damage on the basis of longitudinal arthroscopic observations.

In both the progression and non-progression groups, arthroscopic evaluations of morphological cartilage damage at baseline revealed similar numbers of grade $0-I V$ lesions (Table 2). In fact, morphologically normal cartilage and minimally damaged lesions (grade $0-\mathrm{I}$ ) exhibited onset and progression of cartilage damage, respectively, in the progression group (Table 2). Interestingly, cut-off values of $55.7 \mathrm{nmol} / \mathrm{ml}$ for $\Delta \mathrm{di}-\mathrm{C} 6 \mathrm{~S}, 10.6 \mu \mathrm{g} / \mathrm{ml}$ for $\mathrm{KS}$, and 4.6 for $\mathrm{C} 6 \mathrm{~S} / \mathrm{C} 4 \mathrm{~S}$, as determined by ROC analysis (Fig. 2), correspond to grades 2-4 according to the Kellgren-Lawrence grading scale [6, 10, 24], or advanced OA [25]. This suggests that those showing progression of high-grade cartilage damage exhibited a cartilage aggrecan metabolism similar to that observed in patients with advanced radiographic OA [12], although no radiographic OA changes were observed in this study. Therefore, cut-off values of $\Delta \mathrm{di}-\mathrm{C} 6 \mathrm{~S}, \mathrm{KS}$, and $\mathrm{C6S} / \mathrm{C} 4 \mathrm{~S}$ could be used as biomarkers to predict onset/progression of cartilage damage. Changes in proteoglycans, almost certainly released primarily from aggrecan because of its much greater content of these glycosaminoglycans, reflect cartilage metabolism at an early stage of OA [26]. Although the loss of aggrecan leads to increased aggrecan synthesis, newly synthesized molecules are composed of enriched C4S instead of C6S and KS [8-10], unlike those found in normal SF [16]. Our results are consistent with previous reports $[8-10,16]$, in that levels of $\triangle \mathrm{di}-\mathrm{C} 6 \mathrm{~S}$ and $\mathrm{KS}$ and the ratio of C6S/ C4S were negatively correlated with cartilage damage progression.

Table 3 Biomarker levels of patients in the progression and non-progression groups at ACL reconstruction (baseline)

\begin{tabular}{|c|c|c|c|c|c|}
\hline Variables & & Total $(n=62)$ & Progression group $(n=15)$ & Non-progression group $(n=47)$ & $p$ value \\
\hline $\mathrm{C} 2 \mathrm{C}(\mathrm{ng} / \mathrm{ml})$ & Median (IQR) & $9.8(5.8-13.8)$ & $8.8(5.6-11.8)$ & $10.4(6.1-14.3)$ & 0.282 \\
\hline$\Delta \mathrm{di}-\mathrm{C} 6 \mathrm{~S}(\mathrm{nmol} / \mathrm{ml})$ & Median (IQR) & $69.6(54.9-95.1)$ & $53.4(50.9-69.4)$ & $73.5(60.7-99.9)$ & 0.004 \\
\hline$\Delta \mathrm{di}-\mathrm{C} 4 \mathrm{~S}(\mathrm{nmol} / \mathrm{ml})$ & Median (IQR) & $17.1(14.2-21.6)$ & $16.3(14.8-17.8)$ & $18.0(14.2-23.0)$ & 0.170 \\
\hline $\mathrm{KS}(\mu \mathrm{g} / \mathrm{ml})$ & Median (IQR) & $11.6(9.4-15.6)$ & $9.9(8.3-11.4)$ & $11.9(10.0-16.0)$ & 0.021 \\
\hline $\mathrm{C} 2 \mathrm{C} / \mathrm{KS}$ ratio & Median (IQR) & $0.71(0.51-1.2)$ & $0.67(0.52-1.2)$ & $0.75(0.51-1.2)$ & 0.928 \\
\hline C6S/C4S ratio & Median (IQR) & $4.2(3.6-4.9)$ & $3.9(3.2-4.4)$ & $4.4(3.7-5.2)$ & 0.028 \\
\hline
\end{tabular}

Progression group, patients with an increase (between baseline and follow-up) in the number of high-grade cartilage lesions (Outerbridge grades III and IV) by one score in any of the six compartments; Non-progression group, patients with no increase in the number of high-grade cartilage lesions; IQR interquartile range. $P<0.05$ was considered statistically significant 

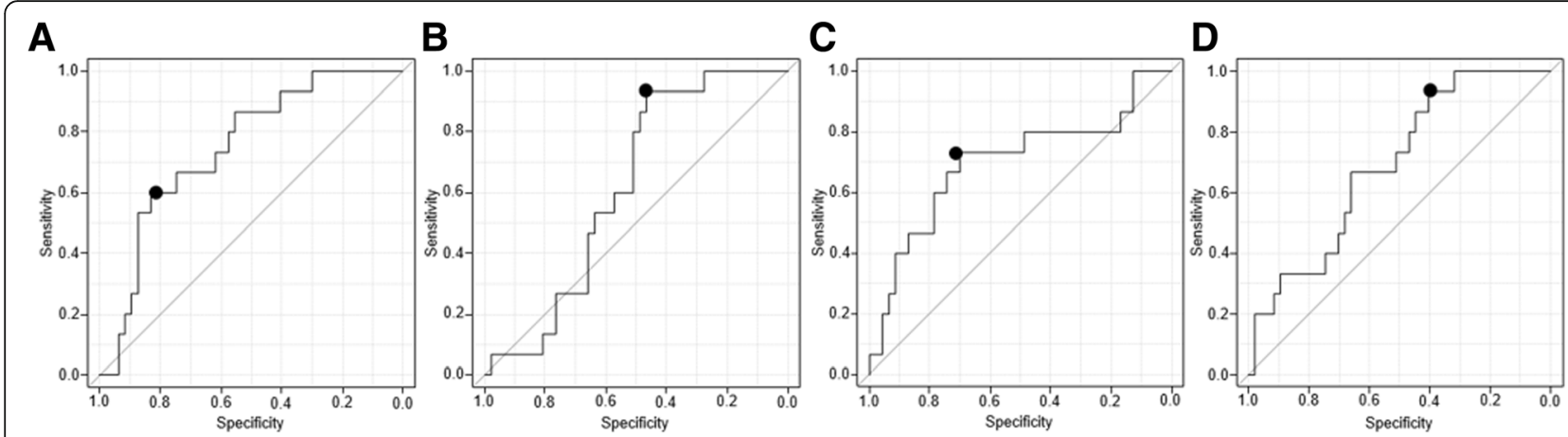

Fig. 2 ROC curves for $\Delta$ di-C6S level, $\Delta$ di-C4S level, KS level, C6S/C4S, and the presence of high-grade cartilage progression. Black dots in the figure panels indicate cut-off points. a ROC analysis for the level of $\triangle$ di-C6S and the presence of high-grade cartilage progression (area under the ROC curve: 0.746 [95\% Cl $0.612-0.880]$; $60.0 \%$ sensitivity and $83.0 \%$ specificity for a cut-off of $55.7 \mathrm{nmol} / \mathrm{ml})$. b ROC analysis for the level of $\Delta$ di-C4S and the presence of high-grade cartilage progression (area under the ROC curve 0.618 [95\% Cl 0.478-0.758]; $93.3 \%$ sensitivity and $46.8 \%$ specificity for a cut-off of $19.0 \mathrm{nmol} / \mathrm{ml})$. c ROC analysis for the level of KS and the presence of high-grade cartilage progression (area under the ROC curve: 0.699 [95\% Cl 0.526-0.872]; $73.3 \%$ sensitivity and $70.2 \%$ specificity for a cut-off of $10.6 \mu \mathrm{g} / \mathrm{ml}$ ). $\mathbf{d}$ ROC analysis for C6S/C4S (ratio of $\Delta$ di-C6S to $\Delta \mathrm{di}-\mathrm{C} 4 \mathrm{~S}$ ) and the presence of high-grade cartilage progression (area under the ROC curve: 0.689 [95\% Cl 0.546-0.833]; $93.3 \%$ sensitivity and $40.0 \%$ specificity for a cut-off of 4.6)

With regard to $\Delta$ di-C4S, when this was added to the logistic regression analysis, a significant difference was noted such that progression of cartilage damage did not advance if $\Delta \mathrm{di}-\mathrm{C} 4 \mathrm{~S}$ was above $19.0 \mathrm{nmol} / \mathrm{ml}$ (calculated from the ROC curve) (Table 4), although a significant difference was not found between the progression group and non-progression group (Table 3), and the area under the ROC curve for $\triangle \mathrm{di}-\mathrm{C} 4 \mathrm{~S}$ was 0.618 , which was lower than that for $\Delta$ di-C6S (0.746), KS (0.699), and C6S/C4S (0.689) (Fig. 2). Normally, $\Delta$ di-C4S is highly expressed in OA cartilage [8-10], which seems to contradict the result of this study. However, $\triangle \mathrm{di}-\mathrm{C} 4 \mathrm{~S}$ and $\Delta \mathrm{di}-\mathrm{C} 6 \mathrm{~S}$ showed a significant positive correlation (correlation coefficient $=0.838 \quad$ [95\% CI 0.744-0.899, $p=0]$ ) with Pearson's product-moment correlation in this study. We surmise that, with regard to early changes following trauma, as long as synthesis of $\triangle \mathrm{di}-\mathrm{C} 6 \mathrm{~S}$ was greater than that of $\Delta$ di-C4S, progression of cartilage damage could not advance. Therefore, it is appropriate to consider also the ratio of $\mathrm{C} 6 \mathrm{~S} / \mathrm{C} 4 \mathrm{~S}$ as a predictive biomarker for progression of cartilage damage.

Our previous cross-sectional study found that $\mathrm{C} 2 \mathrm{C}$, which reflects increased collagenase cleavage of cartilage type II collagen, was significantly associated with the presence of arthroscopic high-grade cartilage damage [1]. C2C is considered a sensitive marker for detecting early cartilage damage. However, in the present longitudinal study, $\mathrm{C} 2 \mathrm{C}$ was not associated with the onset/progression of high-grade cartilage damage. Mean SF C2C level in OA patients $(\mathrm{n}=54)$ was reported to be $30.5 \mathrm{ng} /$ $\mathrm{ml}$ in a previous study [27], whereas the median $\mathrm{C} 2 \mathrm{C}$ level in the present study was $9.8 \mathrm{ng} / \mathrm{ml}$ (interquartile range, 5.8-13.8). Since our patients were at the preradiographic $\mathrm{OA}$ stage, their $\mathrm{C} 2 \mathrm{C}$ levels might have been lower than those of OA patients and thus did not predict the progression of cartilage damage. In a recent

Table 4 Odds ratios for the presence of high-grade cartilage damage progression by $\Delta$ di-C6S, KS levels, C6S/C4S

\begin{tabular}{|c|c|c|c|c|c|c|c|}
\hline \multirow[t]{2}{*}{ Variables } & & \multicolumn{3}{|c|}{ Unadjusted OR } & \multicolumn{3}{|c|}{ Adjusted OR } \\
\hline & & & $(95 \% \mathrm{Cl})$ & $p$ value & & $(95 \% \mathrm{Cl})$ & $\overline{p \text { value }}$ \\
\hline$\Delta \mathrm{di}-\mathrm{C6S}$ & $\leq 55.7$ & 1 & Reference & & 1 & Reference & \\
\hline$(\mathrm{nmol} / \mathrm{ml})$ & $>55.7$ & 0.179 & $(0.051-0.638)$ & 0.008 & 0.231 & $(0.061-0.879)$ & 0.032 \\
\hline$\Delta \mathrm{di}-\mathrm{C} 4 \mathrm{~S}$ & $\leq 19.0$ & 1 & Reference & & 1 & Reference & \\
\hline$(\mathrm{nmol} / \mathrm{ml})$ & $>19.0$ & 0.081 & $(0.010-0.668)$ & 0.020 & 0.081 & $(0.008-0.763)$ & 0.028 \\
\hline KS & $\leq 10.6$ & 1 & Reference & & 1 & Reference & \\
\hline$(\mu \mathrm{g} / \mathrm{ml})$ & $>10.6$ & 0.154 & $(0.042-0.568)$ & 0.005 & 0.114 & $(0.024-0.529)$ & 0.006 \\
\hline \multirow[t]{2}{*}{$\mathrm{C} 6 \mathrm{~S} / \mathrm{C} 4 \mathrm{~S}$} & $\leq 4.6$ & 1 & Reference & & 1 & Reference & \\
\hline & $>4.6$ & 0.105 & $(0.013-0.869)$ & 0.037 & 0.060 & $(0.005-0.737)$ & 0.028 \\
\hline
\end{tabular}

All independent variables were dichotomized using the cut-off points calculated by ROC analysis and tested in a binary manner. The adjusted model included variables (age, duration from injury to $\mathrm{ACL}$ reconstruction, sex, and the number of high-grade cartilage lesions [Outerbridge grades III and IV]) at baseline. $P<0.05$ was considered statistically significant 
study, a C2C-HUSA urine assay, which measures a more specific degradation product(s), revealed the association between baseline data and knee OA progression [18].

Older patients reportedly have increased cartilage damage and more severe OA changes after ACL injury [28]. Age is negatively correlated with the ratio of C6S/ $\mathrm{C} 4 \mathrm{~S}$ [15], and the $\mathrm{C} 6 \mathrm{~S} / \mathrm{C} 4 \mathrm{~S}$ ratio is typically lower in females than in males [15]. This is likely due to the decreased cartilage repair capacity in older patients, and may even reflect gender specificity. In a previous study, we found that both age and duration from injury to first surgery were positively correlated with the number of high-grade cartilage lesions [1]. Moreover, mean age and mean duration were higher in the progression group compared to the non-progression group (Table 1). In addition, baseline cartilage damage might be an important driver for cartilage damage progression. Thus, age, duration from injury to first surgery, sex, and the number of high-grade lesions (grades III and IV) at baseline should all be taken into account when predicting cartilage damage. As shown in Table 4, the levels of $\triangle \mathrm{di}-\mathrm{C} 6 \mathrm{~S}$ and $\mathrm{KS}$ and the ratio of $\mathrm{C} 6 \mathrm{~S} / \mathrm{C} 4 \mathrm{~S}$ were associated with the progression of high-grade cartilage damage, even after adjusting for age, duration from injury to first surgery, sex, and the number of high-grade lesions (grades III and IV) at baseline in multivariable logistic regression analyses. Therefore, levels of $\Delta \mathrm{di}-\mathrm{C} 6 \mathrm{~S}$ and $\mathrm{KS}$ and the $\mathrm{C6S} / \mathrm{C} 4 \mathrm{~S}$ ratio could serve as meaningful predictive biomarkers.

Meniscal tears or defects are risk factors for knee OA [29]. However, the proportion of high-grade meniscus damage in the progression group was similar to that in the non-progression group, at both the first and second evaluations (Table 1). The main component of the meniscus is type I collagen. In contrast to articular cartilage that mainly comprises type II collagen and contains abundant proteoglycans, the meniscus is highly deficient in the proteoglycans [30]. In this regard, meniscus damage is unlikely to contribute to differences in the biomarkers in both groups.

Whether ACL reconstruction performed at our institute is reproducible or not is of considerable importance. All ACL reconstructions were performed by three senior surgeons who had operated on more than 100 cases. Clinical results of ACL reconstruction and arthroscopic longitudinal changes of cartilage damage after ACL reconstruction in this study were comparable to those of previous reports [31, 32].

There are some limitations to this study. First, only a limited number of biomarkers were evaluated. Various biomarkers that reflect cartilage metabolism are available [33], including procollagen II C-propeptide (CPII) [34], which serves as an indicator of collagen synthesis. Indeed, the progression of OA might reflect collagen synthesis rather than collagen cleavage [35]. Thus, changes in collagen metabolism in the early stages of OA should be explored. Second, as this study was conducted retrospectively, the duration from the first to second evaluation varied by patient. That said, the duration was approximately 2 years in most patients in both groups, and given the lack of significant differences between the two groups, its influence was likely minimal. Third, the sample size is relatively small for evaluation of predictive biomarkers. Thus, a bigger group will be needed to demonstrate whether the obtained cut-off values are proper for prediction of cartilage damage progression. Finally, the present study examined cartilage damage after ACL injury based on longitudinal arthroscopic observation, but did not address whether cartilage damage will progress further to radiographic OA damage. A longer observation period would be required to address this issue.

\section{Conclusions}

Progression of high-grade cartilage damage as evaluated by arthroscopic evaluation was significantly associated with biomarkers of aggrecan glycosaminoglycan metabolism; namely, levels of $\triangle \mathrm{di}-\mathrm{C} 6 \mathrm{~S}$ and $\mathrm{KS}$ and the ratio of $\Delta$ di-C6S to $\Delta$ di-C4S. The cut-off values determined in this study may be useful for predicting the progression of cartilage damage.

\section{Additional files}

Additional file 1: Supplementary data for patient baseline characteristics of 62 patients in the current study and 46 patients who dropped out of our follow-up study. (XLS $30 \mathrm{~kb}$ )

Additional file 2: Datasets generated during the current study. (CSV 11 kb)

\section{Abbreviations}

ACL: Anterior cruciate ligament; C2C: Collagenase-generated cleavage neoepitope of type II collagen; KS: Keratan sulfate; OA: Osteoarthritis; SF: Synovial fluid; $\Delta$ di-C4S: Chondroitin-4-sulfate; $\Delta$ di-C6S: Chondroitin-6sulfate

\section{Acknowledgements}

We thank Ms. Izumi Unno for her technical assistance with the immunoassays.

\section{Funding}

This work was funded by Grants-in-Aid for Scientific Research from the Japan Society for the Promotion of Science to TK (Project number: 26462290).

Availability of data and materials

Datasets generated during the current study are available in Additional file 2.

Authors' contributions

TK and YS contributed to the conception and design of the study, acquisition of data, analysis and interpretation of data, drafting/revising the manuscript critically for important intellectual content, and provided final approval of the version to be submitted. KK, ST, and HY contributed to the conception and design of the study, acquisition of data, revising the manuscript critically for important intellectual content, and provided final approval of the version to be submitted. $\mathrm{NI}$ contributed to the conception 
and design of the study, analysis and interpretation of data, revising the manuscript critically for important intellectual content, and provided final approval of the version to be submitted. RP contributed to the use of biomarkers in the study, interpretation of the results, and the writing of the manuscript. All authors read and approved the final manuscript.

\section{Ethics approval and consent to participate}

The study was approved by the Ethics Committee of Nagoya University School of Medicine (2017-0140). This is retrospective research including the information on synovial fluid obtained from the previous study. Therefore, we disclosed the study information at the site of related facilities instead of using a consent form according to committee procedure.

\section{Consent for publication}

Not applicable

\section{Competing interests}

With the exception of RP, who is a consultant to IBEX Pharmaceuticals (manufacturers of the C2C assay kit) in Montreal, QC, Canada, the authors have no competing interests to report.

\section{Publisher's Note}

Springer Nature remains neutral with regard to jurisdictional claims in published maps and institutional affiliations.

\section{Author details}

${ }^{1}$ Department of Orthopedic Surgery, Nagoya University School of Medicine, 65 Tsurumai, Showa, Nagoya 466-8550, Japan. Orthopedic Surgery, Mitsubishi Nagoya Hospital, 7-8 Sotodoi, Atsuta, Nagoya 456-0013, Japan. ${ }^{3}$ Orthopedic Surgery, Kamiiida Daiichi General Hospital, 2-70 Kamiiidakita, Kita, Nagoya 462-0802, Japan. ${ }^{4}$ Division of Orthopaedics, Department of Surgery, McGill University, Montreal, QC, Canada.

\section{Received: 8 August 2017 Accepted: 13 November 2017} Published online: 06 December 2017

\section{References}

1. Yoshida H, Kojima T, Kurokouchi K, Takahashi S, Hanamura H, Kojima M, et al. Relationship between pre-radiographic cartilage damage following anterior cruciate ligament injury and biomarkers of cartilage turnover in clinical practice: a cross-sectional observational study. Osteoarthritis Cartilage. 2013;21:831-8. doi:10.1016/j.joca.2013.03.009.

2. Nelson F, Billinghurst RC, Pidoux I, Reiner A, Langworthy M, McDermott M, et al. Early post-traumatic osteoarthritis-like changes in human articular cartilage following rupture of the anterior cruciate ligament. Osteoarthritis Cartilage. 2006;14:114-9. doi:10.1016/j.joca.2005.08.005.

3. Price JS, Till SH, Bickerstaff DR, Bayliss MT, Hollander AP. Degradation of cartilage type II collagen precedes the onset of osteoarthritis following anterior cruciate ligament rupture. Arthritis Rheum. 1999;42:2390-8. doi:10. 1002/1529-0131(199911)42:11<2390::AID-ANR18>3.0.CO;2-9.

4. Hollander AP, Heathfield TF, Webber C, Iwata Y, Bourne R, Rorabeck C, et al. Increased damage to type II collagen in osteoarthritic articular cartilage detected by a new immunoassay. J Clin Invest. 1994;93:1722-32. doi:10. 1172/JCl117156.

5. Billinghurst RC, Dahlberg L, lonescu M, Reiner A, Bourne R, Rorabeck C, et al. Enhanced cleavage of type II collagen by collagenases in osteoarthritic articular cartilage. J Clin Invest. 1997;99:1534-45. doi:10.1172/JCl119316.

6. Momohara S, Okada N, Ikari K, Mizuno S, Okamoto H. Dermatan sulfate in the synovial fluid of patients with knee osteoarthritis. Mod Rheumatol. 2007; 17:301-5. doi:10.1007/s10165-007-0594-7.

7. Hardingham T, Bayliss M. Proteoglycans of articular cartilage: changes in aging and in joint disease. Semin Arthritis Rheum. 1990;20:12-33. doi:10. 1016/0049-0172(90)90044-G.

8. Hasegawa M, Nakoshi Y, Tsujii M, Sudo A, Masuda H, Yoshida T, et al. Changes in biochemical markers and prediction of effectiveness of intraarticular hyaluronan in patients with knee osteoarthritis. Osteoarthritis Cartilage. 2008;16:526-9. doi:10.1016/j.joca.2007.09.014.

9. Nakajima A, Nakagawa K, Aoki Y, Sonobe M, Shibata Y, Yamazaki M, et al. Changes in synovial fluid biochemical markers following arthroscopic surgery in patients with knee osteoarthritis. Rheumatol Int. 2013;33:209-14. doi:10.1007/s00296-012-2374-8.
10. Uesaka S, Nakayama Y, Yoshihara K, Ito H. Significance of chondroitin sulfate isomers in the synovial fluid of osteoarthritis patients. J Orthop Sci. 2002;7: 232-7. doi:10.1007/s007760200038.

11. Outerbridge RE. Etiology of chondromalacia patellae. J Bone Jt Surg. 1961; 43:752-7.

12. Kellgren JH, Lawrence JS. Radiological assessment of osteo-arthrosis. Ann Rheum Dis. 1957; 16:494-502.

13. Tegner $Y$, Lysholm J. Rating systems in the evaluation of knee ligament injuries. Clin Orthop Relat Res. 1985;198:43-9. doi:10.1097/00003086198509000-00007.

14. Jakob RP, Stäubli HU, Deland JT. Grading the pivot shift. Objective tests with implications for treatment. J Bone Jt Surg Br. 1987;69:294-9.

15. Uesaka S, Miyazaki K, Ito H. Age-related changes and sex differences in chondroitin sulfate isomers and hyaluronic acid in normal synovial fluid. Mod Rheumatol. 2004;14:470-5. doi:10.1007/s10165-004-0351-0.

16. Yamada H, Miyauchi S, Hotta H, Morita M, Yoshihara Y, Kikuchi T, et al. Levels of chondroitin sulfate isomers in synovial fluid of patients with hip osteoarthritis. J Orthop Sci. 1999;4:250-4.

17. Kanda Y. Investigation of the freely available easy-to-use software "EZR" for medical statistics. Bone Marrow Transplant. 2013;48:452-8. doi:10.1038/bmt. 2012.244.

18. Poole AR, Ha N, Bourdon S, Sayre EC, Guermazi A, Cibere J. Ability of a urine assay of type II collagen cleavage by collagenases to detect early onset and progression of articular cartilage degeneration: results from a populationbased cohort study. J Rheumatol. 2016. doi:10.3899/jrheum.150917.

19. Kraus VB, Collins JE, Hargrove D, Losina E, Nevitt M, Katz JN. Predictive validity of biochemical biomarkers in knee osteoarthritis: data from the FNIH OA Biomarkers Consortium. Ann Rheum Dis. 2017;76(1):186-95. doi:10.1136/ annrheumdis-2016-209252.

20. Berry PA, Maciewicz RA, Wluka AE, Downey-Jones MD, Forbes A, Hellawell $C J$, et al. Relationship of serum markers of cartilage metabolism to imaging and clinical outcome measures of knee joint structure. Ann Rheum Dis. 2010;69:1816-22. doi:10.1136/ard.2009.124420.

21. Tourville TW, Johnson RJ, Slauterbeck JR, Naud S, Beynnon BD. Relationship between markers of type II collagen metabolism and tibiofemoral joint space width changes after ACL injury and reconstruction. Am J Sports Med. 2013;41:779-87. doi:10.1177/0363546513476481.

22. Huebner JL, Bay-Jensen AC, Huffman KM, He Y, Leeming DJ, McDaniel GE, et al. Alpha C-telopeptide of type I collagen is associated with subchondral bone turnover and predicts progression of joint space narrowing and osteophytes in osteoarthritis. Arthritis Rheumatol. 2014;66:2440-9. doi:10.1002/art.38739.

23. Saberi Hosnijeh F, Siebuhr AS, Uitterlinden AG, Oei EHG, Hofman A, Karsdal MA, et al. Association between biomarkers of tissue inflammation and progression of osteoarthritis: evidence from the Rotterdam study cohort. Arthritis Res Ther. 2016;18(1):81. doi:10.1186/s13075-016-0976-3.

24. Uesaka S, Nakayama Y, Shirai Y, Yoshihara K. Serum and synovial fluid levels of chondroitin sulfate in patients with osteoarthritis of the knee joint. J Nippon Med Sch. 2001;68:165-70

25. Yamaguchi R, Yamamoto T, Motomura G, Ikemura S, Iwasaki K, Zhao G, et al. Bone and cartilage metabolism markers in synovial fluid of the hip joint with secondary osteoarthritis. Rheumatology (Oxford). 2014;53:2191-5. doi: 10.1093/rheumatology/keu253.

26. Saarakkala S, Julkunen P, Kiviranta P, Makitalo J, Jurvelin JS, Korhonen RK Depth-wise progression of osteoarthritis in human articular cartilage: investigation of composition, structure and biomechanics. Osteoarthritis Cartilage. 2010;18:73-81. doi:10.1016/j.joca.2009.08.003.

27. Ishiguro $\mathrm{N}$, Ito $\mathrm{T}$, Oguchi $\mathrm{T}$, Kojima $\mathrm{T}$, Iwata $\mathrm{H}$, lonescu $\mathrm{M}$, et al. Relationships of matrix metalloproteinases and their inhibitors to cartilage proteoglycan and collagen turnover and inflammation as revealed by analyses of synovial fluids from patients with rheumatoid arthritis. Arthritis Rheum. 2001;44: 2503-11. doi:10.1002/1529-0131(200111)44:11<2503::AID-ART430>3.0.CO;2-P.

28. Roos H, Adalberth T, Dahlberg L, Lohmander LS. Osteoarthritis of the knee after injury to the anterior cruciate ligament or meniscus: the influence of time and age. Osteoarthritis Cartilage. 1995;3:261-7. doi:10.1016/S10634584(05)80017-2.

29. Snoeker BA, Bakker EW, Kegel CA, Lucas C. Risk factors for meniscal tears: a systematic review including meta-analysis. J Orthop Sport Phys Ther. 2013: 43:352-67. doi:10.2519/jospt.2013.4295.

30. Sun Y. Histological examination of collagen and proteoglycan changes in osteoarthritic menisci. Open Rheumatol J. 2012;6:24-32. doi:10.2174/ 1874312901206010024. 
31. Gong X, Jiang D, Wang YJ, Wang J, Ao YF, Yu JK. Second-look arthroscopic evaluation of chondral lesions after isolated anterior cruciate ligament reconstruction: single- versus double-bundle reconstruction. Am J Sports Med. 2013;41:2362-7. doi:10.1177/0363546513496064

32. Asano H, Muneta T, Ikeda H, Yagishita K, Kurihara Y, Sekiya I. Arthroscopic evaluation of the articular cartilage after anterior cruciate ligament reconstruction: a short-term prospective study of 105 Patients. Arthroscopy. 2004;20:474-81. doi:10.1016/j.arthro.2004.03.006.

33. Harkey MS, LuC BA, Golightly YM, Thomas AC, Driban JB, Hackney AC, et al. Osteoarthritis-related biomarkers following anterior cruciate ligament injury and reconstruction: a systematic review. Osteoarthritis Cartilage. 2015;23:1 12. doi:10.1016/j.joca.2014.09.004.

34. Svoboda SJ, Harvey TM, Owens BD, Brechue WF, Tarwater PM, Cameron KL. Changes in serum biomarkers of cartilage turnover after anterior cruciate ligament injury. Am J Sport Med. 2013;41:2108-16. doi:10.1177/ 0363546513494180.

35. Cahue S, Sharma L, Dunlop D, Ionescu M, Song J, Lobanok T, et al. The ratio of type II collagen breakdown to synthesis and its relationship with the progression of knee osteoarthritis. Osteoarthritis Cartilage. 2007;15:819-23. doi:10.1016/j.joca.2007.01.016

Submit your next manuscript to BioMed Central and we will help you at every step:

- We accept pre-submission inquiries

- Our selector tool helps you to find the most relevant journal

- We provide round the clock customer support

- Convenient online submission

- Thorough peer review

- Inclusion in PubMed and all major indexing services

- Maximum visibility for your research

Submit your manuscript at www.biomedcentral.com/submit
Biomed Central 